\title{
Continuous stochastic Schrödinger equations and localization
}

\author{
M. Rigo, F. Mota-Furtado and P.F. O'Mahony \\ Department of Mathematics, Royal Holloway, University of London, Egham, Surrey \\ TW20 0EX, UK
}

\begin{abstract}
The set of continuous norm-preserving stochastic Schrödinger equations associated with the Lindblad master equation is introduced. This set is used to describe the localization properties of the state vector toward eigenstates of the environment operator. Particular focus is placed on determining the stochastic equation which exhibits the highest rate of localization for wide open systems. An equation having such a property is proposed in the case of a single non-hermitian environment operator. This result is relevant to numerical simulations of quantum trajectories where localization properties are used to reduce the number of basis states needed to represent the system state, and thereby increase the speed of calculation.
\end{abstract}

\section{Introduction}

A quantum system interacting with its environment can be described, in the Markovian approximation, by two complementary approaches. In the first and most commonly used [1, 2, 3], the system is represented by a density operator and its evolution is described by a master equation. In the second a state vector represents the system and a stochastic Schrödinger equation describes the state evolution [4, 5, 6] These two treatments are equivalent in the following sense: for all times an ensemble of state vectors generated by a stochastic Schrödinger equation reproduces, on average, the density operator generated by the master equation. The correspondence is not uniquely defined, in that for a single master equation there are many associated stochastic equations.

Stochastic state vector equations, also called unravelings of the master equation, have been introduced in different contexts and with different interpretations. In the fundamental theory of quantum measurement, stochastic equations have been used to describe the general dynamical process of the state collapse into an eigenstate of the measured observable, i.e. localization [1, 8, 8, 9, 10, 11, 12, 13. In quantum

optics, stochastic Schrödinger equations have been used to describe the system state conditioned by measurement outcomes. In this context, an unraveling corresponds to 
a specified measurement scheme, such as photon counting, heterodyne or homodyne detection [6, 14]. More generally, in the field of open quantum systems, unravelings have been used as an efficient numerical method to solve the master equation 15, 16, 17, 18, 19, 20, 21, 22, 23, 24, 25, 26].

The present work is motivated by a recent study of a quantum system in interaction with a thermal bath using the quantum jump (QJ) unraveling [27]. It is shown that, under some assumptions valid in the classical limit, the QJ trajectories, i.e., the realization of the stochastic process, approach a diffusive limit very similar to the one exhibited by the quantum state diffusion (QSD) trajectories. Since diffusion is expected to be a general feature associated with the emergence of classicality, a description of the whole set of continuous unravelings becomes important. This set is introduced in a unified way in the following section. We will show that each continuous unraveling can be characterized very simply by specifying its noise correlations. The set of continuous unravelings is then used to study how quantum state properties, such as localization, evolve when the unraveling changes.

A very important characteristic of quantum trajectories which has both physical and numerical consequences is the localization of quantum states toward eigenstates of the environment operator. Working with the real noise unraveling (RN), Gisin [7] has shown that for an arbitrary hermitian Lindblad operator $L$ the state vectors concentrate on the eigenspace of $L$. Percival [9] extended this result by giving a proper definition of the ensemble localization of an arbitrary operator and then providing analytical bounds for the rate of self-localization of hermitian and nonhermitian Lindblad operators for the quantum state diffusion unraveling. For a dissipative interaction, Garraway and Knight [28, 29] have presented numerical simulations of the localization process using the QJ and QSD unravelings. Starting from different quantum states, such as a superposition of two coherent states, a Fock state and a squeezed ground state, they have shown that such states are highly sensitive to dissipation. They also illustrated the localization process. Recently they have applied their results to describe the evolution of a Schrödinger cat state in a Kerr medium where localization competes with nonlinear effects [30] (see also [31).

For numerical simulation of open quantum systems, individual trajectories have proven advantageous over density operator computations. The main advantages stem from the fact that less space is needed to store and propagate in time a state vector than a density matrix. In addition, for trajectory methods one can exploit the localization property to reduce the number of basis states needed to represent the state vector, thus significantly reducing the time needed to calculate quantum trajectories. For quantum jump unravelings, when many Lindblad operators are present, it is well known that one can perform a unitary transformation to select one of the quantum jumps unravelings, in such a way as to minimize the number of basis states needed (see ref. [22] for an 
application of this property). The localization of the state vector for QSD has been exploited in the mixed representation of Steimle and al. 23] and the moving basis of Schack and al. [24, 25, 26].

In section 3 we use the set of continuous unravelings to describe localization properties for a single environment operator. Several well known results are recovered for a hermitian operator [4, 32]. In the case of a non-hermitian operator the minimal rate of localization introduced for the QSD unraveling [9, 11, 12] is extended to the complete set showing that localization is a general feature shared by all the continuous unravelings, and a new unraveling is introduced. Some theoretical arguments supported by numerical simulations suggest that this new unraveling possesses the highest localization rate.

In the present work we make use of the freedom of choice for the noise correlations to obtain the continuous unraveling which localizes the state vector the most. This transformation should not be confused with the unitary transformation discussed above. These two transformations are complementary and can be used together. At the end of section 3 we compare the localization properties of QSD and of our proposed unraveling.

Finally, in section 1 we summarize our results and draw conclusions about the applicability of the set of continuous unravelings to the study of the quasi-classical limit of open quantum systems.

\section{Continuous unravelings}

We proceed following closely the derivation of the quantum state diffusion unraveling by Gisin and Percival [8]. In this work, a general stochastic differential equation with a complex Wiener process is used as a starting point. The drift and noise terms are then specified by asking that the stochastic differential equation recovers on average the Lindblad master equation for the density operator $\rho$ of the system

$$
\dot{\rho}=-\frac{i}{\hbar}[H, \rho]+\sum_{j=1}^{J}\left(L_{j} \rho L_{j}^{\dagger}-\frac{1}{2}\left\{L_{j}^{\dagger} L_{j}, \rho\right\}\right),
$$

where $H$ is the system Hamiltonian and $L_{j}(j=1, \ldots, J)$ the set of Lindblad operators which represent the influence of the environment. (Since the master equation (11) is valid under a Markovian approximation, all the stochastic differential equations considered apply only within this approximation). The other conditions needed to specify the drift and noise terms are that the state remains normalized and that the stochastic equation shares the same invariance properties under unitary transformations as the master equation. This last constraint is used to prove the uniqueness of QSD among the set of continuous unravelings. Removing the constraint of invariance under unitary transformations among the Lindblad operators, we obtain the set of continuous normpreserving unravelings related to the master equation. 


\subsection{Derivation of the stochastic Schrödinger equations}

We start by considering a general stochastic differential equation of the following Itô form which gives the variation $|d \psi\rangle$ of the state vector $|\psi\rangle$ in a time $d t$

$$
|d \psi\rangle=|v\rangle d t+\sum_{j=1}^{J}\left|u_{j}\right\rangle\left(\sum_{n=1}^{N} \alpha_{j n} d W_{j n}\right)
$$

where $|v\rangle$ and $\left|u_{j}\right\rangle$ are vectors, $\alpha_{j n}$ are complex numbers and $d W_{j n}$ independent real Wiener processes [33] which obey the following relationships

$$
M\left(d W_{j n}\right)=0 \quad d W_{j n} d W_{k m}=\delta_{j k} \delta_{m n} d t \quad d W_{j n} d t=0
$$

where $M$ represents the ensemble average. The two conditions to be respected by the previous equation (2) are (i) the state is normalized for all times $\langle\psi \mid \psi\rangle_{t}=1$ and (ii) for each time, the mean of the projector associated to the state $|\psi\rangle$ gives the density matrix $\rho=M(|\psi\rangle\langle\psi|)$ with the density matrix $\rho$ evolving according to the master equation in Lindblad form (1). In the following, these two conditions will be used to relate the drift term $|v\rangle$ and the stochastic term $\left|u_{j}\right\rangle$ to the state $|\psi\rangle$ as well as giving constraints on the complex numbers $\alpha_{j n}$. Notice that the $\alpha_{j n}$ may also depend on the state $|\psi\rangle$ and on time $t$.

By following closely the QSD derivation given in reference [8], we obtain the expression for the drift term

$$
|v\rangle=-\frac{i}{\hbar} H|\psi\rangle-\frac{1}{2} \sum_{j}\left(L_{j}^{\dagger} L_{j}+\left\langle L_{j}^{\dagger}\right\rangle_{\psi}\left\langle L_{j}\right\rangle_{\psi}-2\left\langle L_{j}^{\dagger}\right\rangle_{\psi} L_{j}\right)|\psi\rangle
$$

where $\left\langle L_{j}\right\rangle_{\psi}=\left\langle\psi\left|L_{j}\right| \psi\right\rangle$ is the expectation value of $L_{j}$ for the state $|\psi\rangle$. The drift term $|v\rangle$ is the same as that obtained in the QSD derivation, but the stochastic vectors $\left|u_{j}\right\rangle$ become

$$
\left|u_{j}\right\rangle=\frac{1}{\sqrt{\sum_{n}\left|\alpha_{j n}\right|^{2}}} \sum_{k} \beta_{j k}\left(L_{k}-\left\langle L_{k}\right\rangle_{\psi}\right)|\psi\rangle \quad j=1 \ldots J
$$

which differs from the QSD derivation by the introduction of the normalization factor $\left(\sum_{n}\left|\alpha_{j n}\right|^{2}\right)^{-1 / 2}$ and the set of complex numbers $\beta_{j k}$. The latter are arbitrary coefficients of a $J \times J$ unitary matrix which arises due to the freedom of choice in the linear combination of vectors $\left(L_{k}-\left\langle L_{k}\right\rangle_{\psi}\right)|\psi\rangle$ used to express $\left|u_{j}\right\rangle$.

Finally one gets the equation for the state vector increment

$$
\begin{aligned}
|d \psi\rangle= & -\frac{i}{\hbar} H|\psi\rangle d t-\frac{1}{2} \sum_{j=1}^{J}\left(L_{j}^{\dagger} L_{j}+\left\langle L_{j}^{\dagger}\right\rangle_{\psi}\left\langle L_{j}\right\rangle_{\psi}-2\left\langle L_{j}^{\dagger}\right\rangle_{\psi} L_{j}\right)|\psi\rangle d t \\
& +\sum_{k=1}^{J}\left(L_{k}-\left\langle L_{k}\right\rangle_{\psi}\right)|\psi\rangle d \zeta_{k}
\end{aligned}
$$


This equation shows that all the indeterminacy due to the coefficients $\alpha_{j n}$ and to the unitary transformation $\left(\beta_{j k}\right)$ can be included in the noise terms $d \zeta_{j}$ which are given by

$$
d \zeta_{k}=\sum_{j=1}^{J} \beta_{j k} \frac{\sum_{n} \alpha_{j n} d W_{j n}}{\sqrt{\sum_{n}\left|\alpha_{j n}\right|^{2}}}
$$

It can be seen easily that they have zero mean $M\left(d \zeta_{j}\right)=0$ and correlations

$$
d \zeta_{j} d \zeta_{k}^{*}=\delta_{j k} d t \quad \text { and } \quad d \zeta_{j} d \zeta_{k}=c_{j k} d t
$$

where $c_{j k}$ are correlation coefficients related to the unitary transformation $\left(\beta_{j k}\right)$ and the noise coefficients $\alpha_{i n}$ in the following way

$$
c_{j k}=\sum_{i=1}^{J} \beta_{i j} \beta_{i k} c_{i} \quad \text { with } \quad c_{i}=\frac{\sum_{n} \alpha_{i n}^{2}}{\sum_{n}\left|\alpha_{i n}\right|^{2}}
$$

and $c_{i}$ are complex numbers inside the unit circle $\left|c_{i}\right| \leq 1$ for all $i=1, \ldots, J$ (see Appendix). Notice that although the stochastic process is completely specified by the numbers $\beta_{j k}$ and $\alpha_{i n}$, that specification will be non-unique in that all sets of numbers that yield the same correlation coefficients $c_{j k}$ through equation (9) will describe the same stochastic process [33]. Thus an unraveling is completely specified when the correlation coefficients $c_{j k}$ are given. This result provides a natural classification for continuous stochastic Schrödinger equations associated with the completely positive master equation (11). In ref [34 a similar classification is given for all (Markovian) positive master equations but only for two dimensional Hilbert spaces. This suggests that the same classification procedure can be extended to every positive master equation in arbitrary dimensions.

In the absence of a unitary transformation $\left(\beta_{j k}=\delta_{j k}\right)$ and with only one Wiener process $N=1$, the complex noises $d \zeta_{j}$ are necessarily of the form $d \zeta_{j}=e^{i \phi_{j}} d W_{j 1}$. For $\phi_{j}=0$ we recover the real noise unraveling and for $\phi_{j}=\pi / 2$ the imaginary noise unraveling. The present work shows not only that any phase $\phi_{j}$ can be chosen but also that the phase can be a smooth function of the state $|\psi\rangle$ and of the time. The QSD unraveling is recovered taking two Wiener processes $(N=2)$ with $d \zeta_{j}=$ $\left(d W_{j 1}+i d W_{j 2}\right) / \sqrt{2}$ for all $j$. In this special case the correlations $c_{j k}$ vanish.

\subsection{Unitary transformation}

Let us introduce the following unitary transformation among Lindblad operators

$$
L_{j}=\sum_{k} u_{j k} \tilde{L}_{k}-\lambda_{j} \mathbb{1}
$$


where $u_{j k}$ and $\lambda_{j}$ are complex numbers and $\left(u_{j k}\right)$ a unitary matrix [8, 9, 35]. With this transformation the noise terms become $d \tilde{\zeta}_{k}=\sum_{j} u_{j k} d \zeta_{j}$ with the correlations

$$
d \tilde{\zeta}_{j} d \tilde{\zeta}_{k}^{*}=\delta_{j k} d t \quad \text { and } \quad d \tilde{\zeta}_{j} d \tilde{\zeta}_{k}=\sum_{m, n=1}^{J} u_{m j} u_{n k} c_{m n} d t
$$

These correlations will depend on the unitary transformation $\left(u_{j k}\right)$ unless all the correlation factors vanish, i.e. $c_{j k}=0$. Since $\left(\beta_{j k}\right)$ is itself a unitary transformation, a necessary condition for invariance under unitary transformation is given by

$$
c_{j}=0 \quad \text { for all } j=1, \ldots, J
$$

When only one Wiener process $N=1$ is present, the unitary invariance condition (12) implies $\alpha_{j 1}=0$ for all $j$. As a consequence there is no invariant unraveling with only one Wiener process. With two Wiener processes $N=2$, the invariance condition becomes $\alpha_{j 1}^{2}+\alpha_{j 2}^{2}=0$. The norm of the two complex numbers is the same $\left|\alpha_{j 1}\right|=\left|\alpha_{j 2}\right|$ and the phases are related by $\phi_{j 1}-\phi_{j 2}=\pi / 2+n \pi$ where $n$ is any integer number. The simplest case $n=0$ leads to

$$
d \zeta_{j}=e^{i \phi_{j}}\left(\frac{d W_{j 1}+i d W_{j 2}}{\sqrt{2}}\right)
$$

which correspond to the complex noise used in the QSD unraveling when the phases $\phi_{j}$ are set to zero. The simplest case which can satisfy the invariance condition (12) is given by the QSD unraveling. The phases $\phi_{j}$ and other choices of $n$ introduce only irrelevant phase factors which can be neglected. This is the uniqueness result of Gisin and Percival for QSD. If one considers more than two Wiener processes $N \geq 3$, it is possible to construct other unravelings invariant under unitary transformation. For instance:

$$
d \zeta=\frac{d W_{1}+e^{i \pi / 3} d W_{2}+e^{-i \pi / 3} d W_{3}}{\sqrt{3}}
$$

where we have omitted the index $j$ and the phase factor. Since all these unravelings have the same correlations $d \zeta^{2}=0$ and $|d \zeta|^{2}=d t$, they are equivalent [33] and can be replaced by the QSD unraveling.

\section{Localization}

As an application of the set of continuous unraveling obtained in the present work, one can compute the rate of self-localization of a single environment operator $L$ for a wide open system, i.e. $H=0$, and determine the effect of the noise correlation on the rate of self-localization. The rate of self-localization is defined as the rate at which the ensemble average of the quantum mean square deviation decays [9]. It is also the 
ensemble average rate at which the state vector $|\psi\rangle$ tends towards one of the (right) eigenstates of the Lindblad operator. The quantum mean square deviation $\dagger$ of the operator $L$ is defined as $\sigma^{2}(L)=\left\langle L^{\dagger} L\right\rangle_{\psi}-\left\langle L^{\dagger}\right\rangle_{\psi}\langle L\rangle_{\psi}$. More generally the quantum covariance of two operators for the state $|\psi\rangle$ is $\sigma(A, B)=\left\langle A^{\dagger} B\right\rangle_{\psi}-\left\langle A^{\dagger}\right\rangle_{\psi}\langle B\rangle_{\psi}$ [9]. Note that the quantum covariance of $L$ with itself is just the quantum mean square deviation $\sigma^{2}(L)=\sigma(L, L)$. We restrict our attention to a wide open system because we want to describe the localization process, independently of the action of the Hamiltonian. This, clearly, is only a first step towards a proper understanding of localization which should involve Hamiltonian effects as well.

\subsection{Hermitian environment operator}

For a wide open system with a hermitian environment operator $L=L^{\dagger}$ the state vector $|\psi\rangle$ evolves according to

$$
|d \psi\rangle=-\frac{1}{2}\left(L^{\dagger} L+\left\langle L^{\dagger}\right\rangle_{\psi}\langle L\rangle_{\psi}-2\left\langle L^{\dagger}\right\rangle_{\psi} L\right)|\psi\rangle d t+\left(L-\langle L\rangle_{\psi}\right)|\psi\rangle d \zeta
$$

where $d \zeta$ is a noise of the kind previously described by (7) and (8) with an associated correlation factor $c$ given by $d \zeta^{2}=c d t$. When the state evolves according to equation (15) we can compute the change in the expectation value of $L$

$$
d\langle L\rangle_{\psi}=2 \sigma^{2}(L) \operatorname{Re}(d \zeta)
$$

and the change in the quantum mean square deviation

$$
d \sigma^{2}(L)=-2 \sigma^{2}(L)^{2}(1+\operatorname{Re}(c)) d t+2 \sigma\left(L_{\Delta} L_{\Delta}, L\right) \operatorname{Re}(d \zeta)
$$

where we have used the notation $L_{\Delta}=L-\langle L\rangle_{\psi}$. The diffusion of the expectation value $\langle L\rangle_{\psi}$ and the quantum mean square deviation is produced only by the real part of the noise term $d \zeta$.

Taking the ensemble mean shows that the expectation value $M\langle L\rangle_{\psi}=\operatorname{Tr}(\rho L)$ remains constant and the quantum mean square deviation evolves as

$$
M \frac{d \sigma^{2}(L)}{d t}=-2(1+\operatorname{Re}(c)) M\left(\sigma^{2}(L)^{2}\right)
$$

The noise correlation $c$ is a characteristic signature of the chosen unraveling. For $c=0$, the quantum state diffusion result, giving a minimal localization rate of 2 , is recovered [0]. In this case, as in almost all cases, the mean square deviation tends to zero, thus the state $|\psi\rangle$ evolves towards one eigenstate of $L$. The real noise (RN) unraveling $c=1$ is clearly the one which gives the highest rate of self-localization. As a consequence, for numerical simulations involving an arbitrary Hamiltonian and one hermitian environment operator, the RN unraveling should be used since it will produce $\dagger$ Note that the quantum mean square deviation is not an ensemble average. 
the fastest localization (for continuous unravelings). In the opposite case to the RN unraveling, if one uses the imaginary noise unraveling $c=-1$, the mean localization rate vanishes and the state does not evolve towards an eigenstate of $L$. Recovering these well known results [4, 32] confirms the validity of equation (15).

\subsection{Non hermitian environment operator}

We consider the case of a single non hermitian Lindblad operator. Since this case is more difficult to treat, we restrict ourselves to the more specific case of an annihilation operator $L=\sqrt{\kappa} a$. As for a hermitian operator, we want to determine which is the unraveling with the highest localization rate and find out if there is any unraveling which does not localize.

The state vector evolves according to equation (15) and the change in the quantum mean square deviation $\sigma^{2}(a)=\left\langle a^{\dagger} a\right\rangle_{\psi}-\left\langle a^{\dagger}\right\rangle_{\psi}\langle a\rangle_{\psi}$ is given by

$$
\begin{aligned}
d \sigma^{2}(a)= & -\kappa\left(\sigma^{2}(a)+\sigma^{2}(a)^{2}+\left|\sigma\left(a^{\dagger}, a\right)\right|^{2}+2 \sigma^{2}(a) \operatorname{Re}\left\{c \sigma\left(a^{\dagger}, a\right)\right\}\right) d t \\
& +2 \sqrt{\kappa} \operatorname{Re}\left\{\left(\sigma\left(a^{\dagger} a, a\right)-\left\langle a^{\dagger}\right\rangle_{\psi} \sigma\left(a^{\dagger}, a\right)-\langle a\rangle_{\psi} \sigma^{2}(a)\right) d \zeta\right\}
\end{aligned}
$$

which involves the quantum covariance $\sigma\left(a^{\dagger}, a\right)=\left\langle a^{2}\right\rangle_{\psi}-\langle a\rangle_{\psi}^{2}$. The equation for $\sigma\left(a^{\dagger}, a\right)$ can also be derived to give

$$
\begin{aligned}
d \sigma\left(a^{\dagger}, a\right)= & -\kappa\left(\left(1+2 \sigma^{2}(a)\right) \sigma\left(a^{\dagger}, a\right)+c \sigma\left(a^{\dagger}, a\right)^{2}+c^{*} \sigma^{2}(a)^{2}\right) d t \\
& +2 \sqrt{\kappa} \operatorname{Re}\left\{\left(\sigma\left(a^{2}, a\right)-2\left\langle a^{\dagger}\right\rangle_{\psi} \sigma^{2}(a)\right) d \zeta\right\}
\end{aligned}
$$

For the QSD unraveling $c=0$, the two equations (19) and (20) are known to describe the localization of the state $|\psi\rangle$ towards a coherent state in the case of a harmonic oscillator. Furthermore, this localization is known to be globally stable [12.

Taking the ensemble mean over equation (19) removes the noise terms but introduces statistical correlations since the statistical mean of a product is in general different from the product of the statistical means. Thus one cannot obtain an immediate result for the mean rate of localization. However, one can notice that the drift part of $d \sigma^{2}(a)$ can be written as a sum of positive terms:

$$
\sigma^{2}(a)+\left(\sigma^{2}(a)-\left|\sigma\left(a^{\dagger}, a\right)\right|\right)^{2}+2 \sigma^{2}(a)\left|\sigma\left(a^{\dagger}, a\right)\right|\left(1+\operatorname{Re}\left\{c \frac{\sigma\left(a^{\dagger}, a\right)}{\left|\sigma\left(a^{\dagger}, a\right)\right|}\right\}\right)
$$

the third term of this expression being positive since $|c| \leq 1$. As a consequence, the argument for global stability of coherent states,

$$
M \frac{d \sigma^{2}(a)}{d t} \leq-\kappa M \sigma^{2}(a)
$$

is valid for all the continuous unravelings. A localization rate of $\kappa$, associated with the exponential decay of quantum correlations $M \sigma^{2}(a) \simeq \exp (-\kappa t)$, is expected to be 
independent of the choice of unraveling. This result shows that, for an annihilation operator, all unravelings localize and $\kappa$ provides a minimal bound, independent of the unraveling, for the ensemble mean localization rate.

For a hermitian operator, the unraveling which localizes the most was easy to find since the evolution of the quantum mean square deviation is not coupled to any other moment. Furthermore the correlation factor $c$ factorises, making the unraveling independent of the state. In the present case the situation is more complex, since none of these two simplifying conditions are satisfied. In the case of an annihilation operator, we adopt the following technique. Instead of considering the localization of an arbitrary state $|\psi\rangle$, we restrict our attention to squeezed states. We will show that every unraveling (15) with $L=\sqrt{\kappa} a$ preserves the set of squeezed states, i.e., if the initial state is a squeezed state it will evolve into a squeezed state. This has been shown for the QSD unraveling in ref. [12]. The unraveling which reduces the squeezing most efficiently is determined. Finally, some arguments will be given as to why this unraveling should be the one with the highest localization rate for an arbitrary initial state.

Squeezed states are defined as the states $\left|\gamma_{t}, \alpha_{t}\right\rangle$ which satisfy the relation

$$
\left(a-\gamma_{t} a^{\dagger}-\alpha_{t}\right)\left|\gamma_{t}, \alpha_{t}\right\rangle=0
$$

where $\gamma_{t}$ and $\alpha_{t}$ are complex numbers which label the squeezed state $\left|\gamma_{t}, \alpha_{t}\right\rangle$ [36]. When the squeezing parameter $\gamma_{t}$ vanishes, squeezed states reduce to coherent states. Amongst the properties of squeezed states, we will use for our present purposes only the relations between the mean square deviation and the squeezing parameter

$$
\sigma_{s}^{2}(a)=\frac{\left|\gamma_{t}\right|^{2}}{1-\left|\gamma_{t}\right|^{2}}=\gamma_{t} \sigma_{s}\left(a^{\dagger}, a\right)^{*}
$$

where the index $s$ specifies that the mean square deviation is taken with respect to the squeezed state $\left|\gamma_{t}, \alpha_{t}\right\rangle$. This last relation tells us that the mean square deviation depends only on the squeezing parameter.

A condition to check if squeezed states are preserved can be obtained by differentiating (23) [11, 35]. In order to simplify the calculation, the Stratonovich formalism is used. In this formalism, the usual differentiation rules apply. Thus from (23), a state $|\psi\rangle$ initially squeezed will remain squeezed if it is possible to write

$$
\left(a-\gamma_{t} a^{\dagger}-\alpha_{t}\right)|d \psi\rangle=\left(d \gamma_{t} a^{\dagger}+d \alpha_{t}\right)|\psi\rangle
$$

where $|d \psi\rangle$ is to be expressed in Stratonovich form. From equation (15) and using the usual conversion formula $X \circ d Y=X d Y+\frac{1}{2} d X d Y$ between the Stratonovich and Itô formalism [37], one can express the differential increment for the state vector $|\psi\rangle$ as

$$
\begin{aligned}
|d \psi\rangle= & -\frac{1}{2}\left\{L^{\dagger} L-2\left\langle L^{\dagger}\right\rangle_{\psi}\left(L-\langle L\rangle_{\psi}\right)\right\}|\psi\rangle d t \\
& -\frac{c}{2}\left\{L^{2}-\left\langle L^{2}\right\rangle_{\psi}-2\langle L\rangle_{\psi}\left(L-\langle L\rangle_{\psi}\right)\right\}|\psi\rangle d t
\end{aligned}
$$




$$
+\left(L-\langle L\rangle_{\psi}\right)|\psi\rangle \circ d \zeta
$$

Inserting this expression in the condition (25), one finds not only that squeezed states are preserved but also the equations for the squeezing parameters are

$$
\begin{aligned}
& d \gamma_{t}=-\kappa \gamma_{t}\left(1+c \gamma_{t}\right) d t \\
& d \alpha_{t}=-\frac{\kappa}{2} \alpha_{t} d t+\kappa \gamma_{t}\left\langle a^{\dagger}\right\rangle_{s}\left(1+c \gamma_{t}\right) d t+\sqrt{\kappa} \gamma_{t} d \zeta
\end{aligned}
$$

written in Itô form. Since the evolution of the squeezing parameter $\gamma_{t}$ is deterministic, it is easy to find the unraveling which produces the fastest squeezing decay. It is given by the following correlation factor

$$
c=\frac{\gamma_{t}^{*}}{\left|\gamma_{t}\right|}
$$

Notice that this unraveling depends on the state itself. In order to produce the maximal decay in squeezing, the noise term in the stochastic Schrödinger equation has to evolve according to the prescription given in (29). For such an unraveling, the norm of the correlation factor $c$ is kept constant at its maximal value $|c|=1$ and the phase varied in time along each single trajectory in order to minimize the squeezing.

For all the unravelings with a correlation factor having such phase but an arbitrary norm $\left\{c_{r}=r \gamma_{t}^{*} /\left|\gamma_{t}\right|, \quad r \in[0,1]\right\}$, the localization rate can be easily computed. This set includes QSD which as $r=0$ and the adaptive unraveling (29) which has $r=1$. For every unraveling in this set, the squeezing parameter $\gamma_{t}$ decays as $\gamma_{t} \simeq e^{-\kappa t}$ for large enough times. Using the relation (24), one obtains

$$
\sigma^{2}(a) \simeq e^{-2 \kappa t} \quad \sigma\left(a^{\dagger}, a\right) \simeq e^{-\kappa t} \quad \text { for } t \gg \kappa t
$$

Compared to the minimal localization rate of $\kappa$, this result shows that the unravelings which have a correlation factor $c$ with the proper phase, i.e. all unravelings in the above mentioned set, produce a localization rate on squeezed states which is twice the minimal localization rate. Furthermore, the quantum mean square deviation $\sigma\left(a^{\dagger}, a\right)$ decays at the same rate as the energy. Thus, the time needed for a squeezed state to become a coherent state is of the same order as the dissipation time, making the squeezed state a favourite basis for numerical simulations.

Squeezed states are frequently represented in phase space using distribution functions. The $Q$ distribution of a squeezed state is a Gaussian whose contour forms an ellipse. It can be easily seen that the phase (29) of the correlation factor $c$ is equal to $e^{i 2 \phi}$ where $\phi$ is the angle between the real axis and the major axis of the ellipse. The correlation factor $c$ is defined, irrespective of its norm, as the square of the noise term $d \zeta$ (see (8)). Thus the phase of the noise term $d \zeta$, which produces the fastest squeezing decay, is the same as the phase of the major axis of the ellipse, this later being defined as $e^{i \phi}$. One can say that the unraveling varies in time in order to distribute the noise fluctuations along the axis of largest spread of the squeezed state. 
If the system state is not a squeezed state, the previous derivation does not apply anymore. Nevertheless, we can try to generalize the result for an arbitrary state. Using the relation (24) between squeezing parameter and mean quantum deviation, the same unraveling can be specified as

$$
c=\frac{\sigma\left(a^{\dagger}, a\right)^{*}}{\left|\sigma\left(a^{\dagger}, a\right)\right|}
$$

This expression allows us to generalize the criteria obtained for a squeezed state to an arbitrary state. This choice corresponds to fluctuations distributed along the major axis of the ellipse which will give the best approximation to the state distribution in phase space. Another interesting aspect of the unraveling (31) is that it is also the one which maximizes the drift term (21) in the $d \sigma^{2}(a)$ equation. This is not a sufficient condition to insure this unraveling localizes the most since this condition neglects statistical correlations. As soon as statistical correlations can be neglected, this unraveling will produce the highest localization rate.

These two arguments show that for distributions which can be well approximated by ellipses, the unraveling (29) will give the highest localization rate. What happens with other states? To answer this question, numerical simulations have been employed. Using three different initial states, and three different unravelings, the evolution in time of the quantum mean square deviation $\sigma^{2}(a)$ has been computed. The ensemble average $M \sigma^{2}(a)$ taken over 1000 trajectories is represented in figures 내. The initial states chosen are: a Fock state $|24\rangle$, a superposition of Fock states $(|23\rangle+|25\rangle) / \sqrt{2}$ and a superposition of coherent states $(|\alpha\rangle+|-\alpha\rangle) / \sqrt{2}$ with $\alpha=4$. The unravelings chosen are: the unraveling with the proper phase (31), QSD with $c=0$ and real noise with $c=1$.

All our simulations confirm that such quantum states are very unstable under the effect of dissipation [29], this being independent of the chosen unraveling. After a small time $\kappa t<1$, the state becomes a squeezed state to a very good approximation. During the transition from an arbitrary initial state to an almost squeezed state, the rate of localization is very high.

Once the state has become a squeezed state, the mean rate of localization is approximately constant for all the unravelings. The localization rate always lies in between the minimal rate $\kappa$ and the squeezed state rate $2 \kappa$ (see figure 4 ). The latter occurs only for the set of unravelings with the appropriate phase. For the unravelings with a different choice of phase, the term $c \gamma_{t}$ in equation (27) will give a negative contribution and produce a lower localization rate. In all numerical simulations, the new unraveling produced the highest localization.

Finally, one can try to generalize the previous result to an arbitrary Lindblad operator. In this case, the unraveling which localize the most will be the one with 


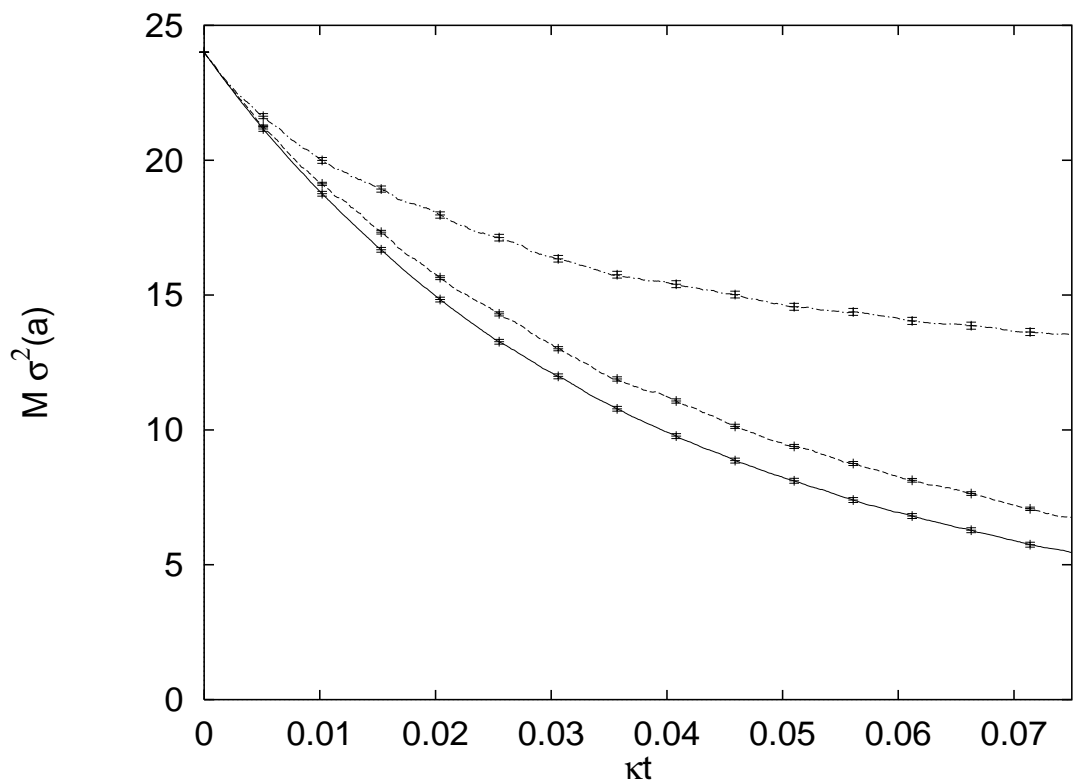

Figure 1. Ensemble average of the quantum mean square deviation $\sigma^{2}(a)$ showing the short time scale localization. The initial state is the Fock state $|24\rangle$. Each curve represent a different unraveling: the unraveling (31) (- - Q), QSD (- - -) and Real Noise (- - - ). The ensemble average is computed using 1000 trajectories. The errors bars indicate the $95 \%$-confidence intervals.

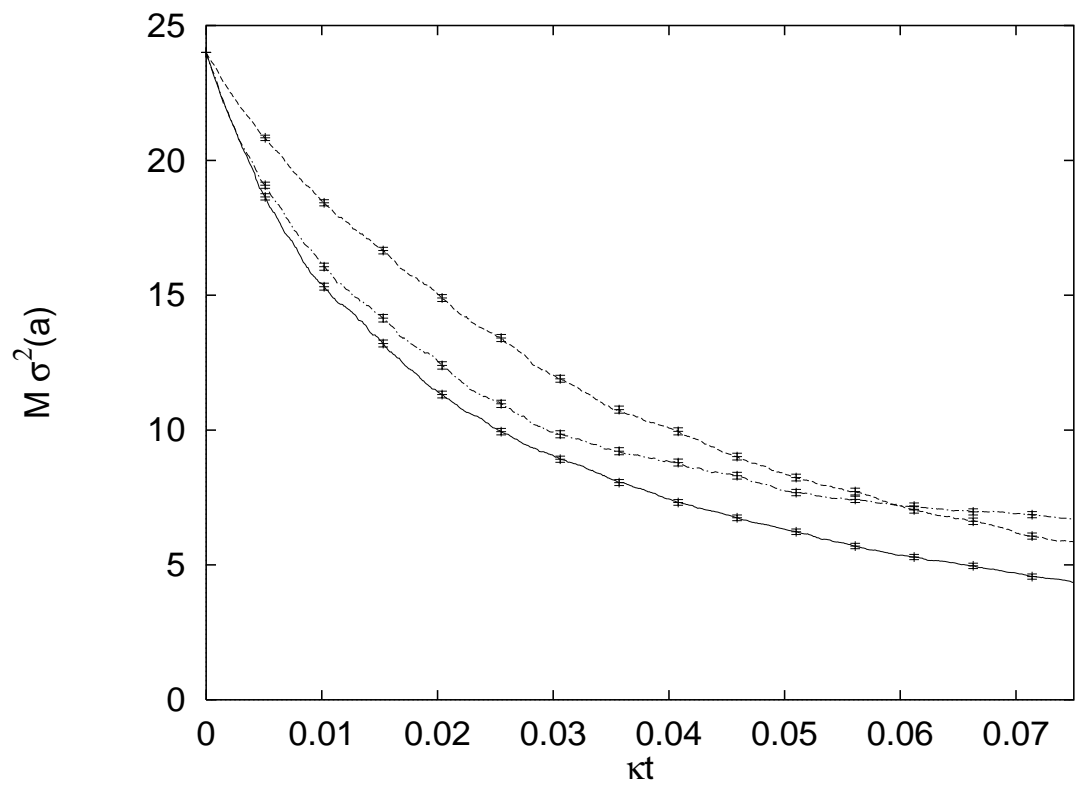

Figure 2. As figure 1, but with the initial state in a superposition of two Fock states $2^{-1 / 2}(|23\rangle+|25\rangle)$. 


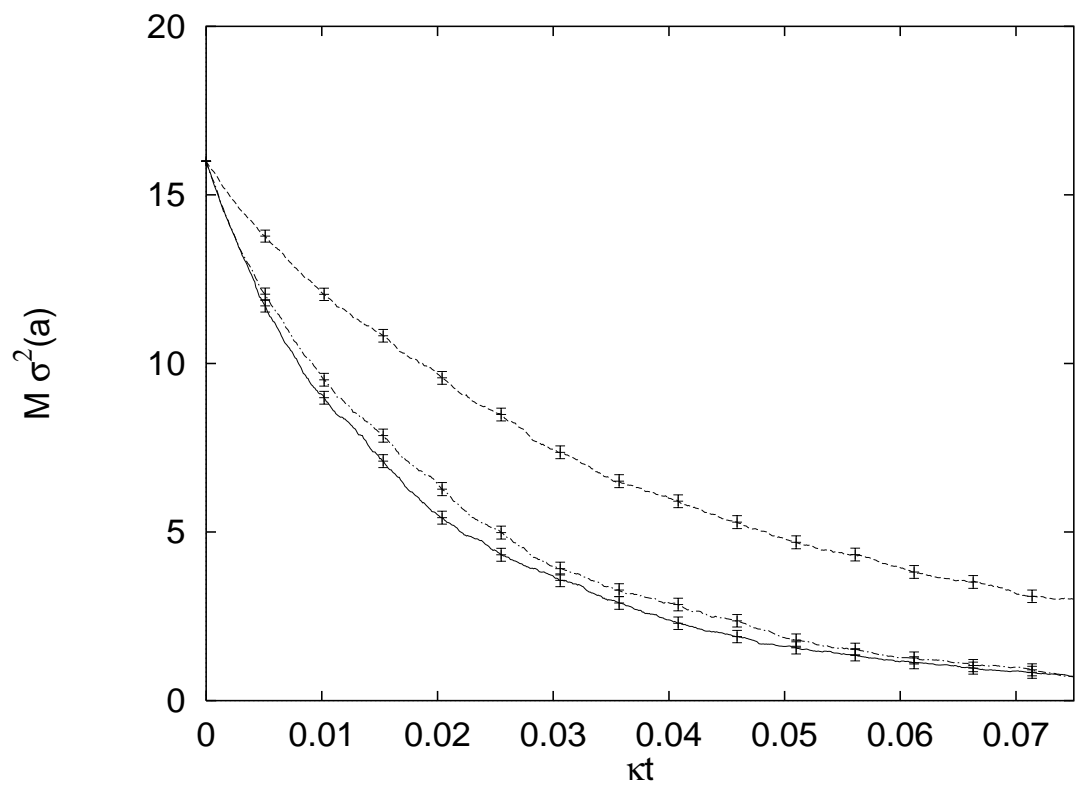

Figure 3. As figure 1, but with the initial state in a superposition of two coherent states $2^{-1 / 2}(|\alpha\rangle+|-\alpha\rangle)$ with $\alpha=4$.

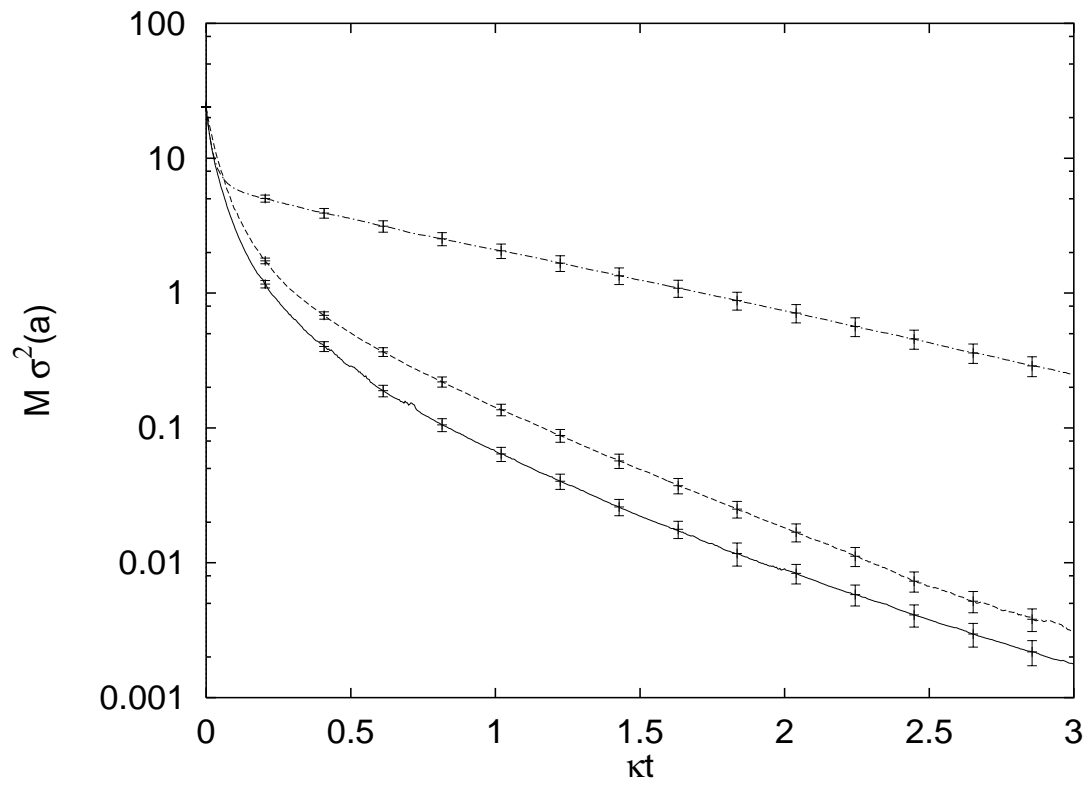

Figure 4. As figure 2, but for a longer time scale. 
a correlation factor given by

$$
c=\frac{\sigma\left(L^{\dagger}, L\right)^{*}}{\left|\sigma\left(L^{\dagger}, L\right)\right|}
$$

For a hermitian operator $q$, this expression reproduces the result previously obtained, namely

$$
c=\frac{\sigma\left(q^{\dagger}, q\right)^{*}}{\left|\sigma\left(q^{\dagger}, q\right)\right|}=\frac{\sigma^{2}(q)}{\left|\sigma^{2}(q)\right|}=1
$$

for the real noise unraveling. For the annihilation operator $L=a$ equation (32) is equivalent to (31).

\subsection{Numerical simulations}

Instead of trying to justify further the unraveling (32) as the most localizing one for an arbitrary Lindblad operator we look for an estimate of the improvement produced by using such an unraveling in numerical simulations. The test consists of computing the quantum mean square deviation $\sigma^{2}(a)$ along a quantum trajectory with both the unraveling (31) and the QSD unraveling. The ensemble average $M \sigma^{2}(a)$ which gives a measure of the size of the wave packet as well as the variance $\operatorname{Var}\left\{\sigma^{2}(a)\right\}=$ $M\left(\sigma^{2}(a)-M \sigma^{2}(a)\right)^{2}$ which measures the fluctuations of the wave packet size are then compared between the two unravelings. The quantity $\sigma^{2}(a)$ is taken here as a measure of the size of the wave packet since it corresponds formally to the excitation number of the state displaced at the origin [27]. For a number states basis, this measure is proportional to the number of basis state needed to represent the state $|\psi\rangle$. The system we used for this test is a kicked anharmonic oscillator

$$
H=i \hbar \beta(t)\left(a^{\dagger}-a\right)+\frac{1}{2} \hbar \chi a^{\dagger 2} a^{2}
$$

subject to dissipation $L=\sqrt{\kappa} a$. The driving $\beta(t)$ is a periodic sequence of rectangular pulses of height $\beta_{0}$, length $\tau_{1}$ and separation $\tau_{2}$. In this case the Hamiltonian can also play an important role in the localization process. This system has been considered in previous publications [38, 39, 35] as a simple example of a chaotic system. In conjunction with this system, the scaling transformations: $\tilde{t}=\lambda t, \tilde{\kappa}=\kappa / \lambda, \tilde{\beta}_{0}=\beta_{0}, \tilde{\chi}=\chi / \lambda^{3}$ have been introduced. This scaling of the parameters does not affect the classical dynamics but when it is introduced in the quantum equations of motion, it allows one to perform the quantum-classical transition, by varying $\lambda$ from $\lambda=1$ (quantum) to $\lambda \rightarrow \infty$ (classical). Here we use this transformation for a different purpose. In the quantum limit, nonlinear effects produce a strong delocalization which can not be compensated by the localization effect of the dissipation. In the classical limit, on the contrary, localization is expected to be dominant. Thus varying the scaling factor allows us to tune the relative strength of the delocalization. 

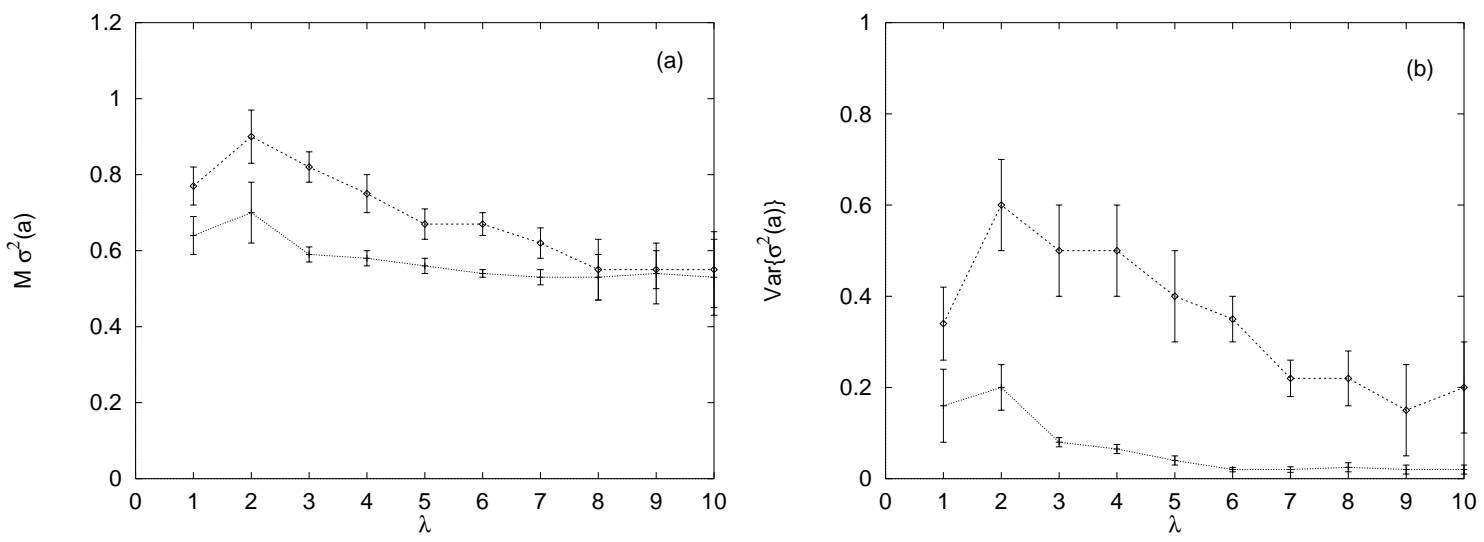

Figure 5. Ensemble average (a) and variance (b) of the quantum mean square deviation $\sigma^{2}(a)$ versus the scaling parameter $\lambda$ for the kicked anharmonic oscillator. The upper curve corresponds to the QSD unraveling and the lower curve to the time dependent unraveling (31). The errors bars take into account statistical as well as numerical accuracy uncertainties.

In figure 5, the ensemble average and variance of the quantum square deviation $\sigma^{2}(a)$ are represented for different values of the scaling parameter $\lambda$. The values represented are stationary results obtained by integrating the equations of motions over typically 2500 periods and taking the mean over a single trajectory. Such a long integration in time is necessary in order to obtain a proper average over the strange attractor of the chaotic system. The system parameters are set to the following values $\chi=1, \beta_{0}=2$, $\kappa=0.5, \tau_{1}=0.98$ and $\tau_{2}=1$ for which the classical system is known to be chaotic. The precision of the numerical results is estimated by repeating the calculations for different numerical parameters such as the time step size.

Figure 5(a) shows for both unravelings a slowly decaying ensemble average $M \sigma^{2}(a)$ for an increasing value of the scaling $\lambda$. Notice that the amplitude of motion rescales as $\lambda$ thus the ratio $\sigma^{2}(a) / M\left\langle a^{\dagger} a\right\rangle$ tends towards zero when $\lambda \rightarrow \infty$, providing a numerical justification for the emergence of the classical attractor. Furthermore, fig 5(a) shows that the unraveling (31) reduces, compared to the QSD unraveling, the stationary value of the mean size of the wave packet. The reduction can be up to $20 \%$ depending on the scale parameter $\lambda$, the largest reduction being achieved in the quantum regime.

More important is the reduction of the size of the fluctuations shown in fig $5(\mathrm{~b})$. The picture suggested is that each time the wave packet deviates from a coherent state the QSD unraveling tends to restore the shape by applying a homogeneous noise, while the unraveling (31) adapts by applying a non-homogeneous noise in the direction of the largest deviation. This adaptability does not produce an important reduction of the wave packet size but can stabilize the wave packet more efficiently as compared to QSD. 


\section{Discussion}

We have introduced the set of continuous unravelings which recovers in mean the master equation in Lindblad form and preserves the norm of the state vector. The quantum state diffusion unraveling is a member of this set, being the simplest which preserves the same invariance properties under unitary transformations as the density matrix. We

have seen that each single unraveling can be specified very simply by the choice of the noise correlations thus providing a natural classification. For theoretical purposes, it is useful to work with the full set of continuous unravelings since it allows one to study how quantities which depend on the choice of the unraveling are sensitive to this choice.

As a first application, we have studied the localization properties when only a single Lindblad operator is present. In the case of a hermitian operator, the highest localization rate of the real noise unraveling as well as the absence of localization of the imaginary noise unraveling have been recovered and explained in a consistent way. For a non hermitian operator, namely the annihilation operator, a new time dependent unraveling has been introduced. It is shown analytically that this unraveling provides the highest localization rate for squeezed states and numerically that this property is also valid for more complex quantum states. This unraveling maximizes the localization by continuously adjusting the phase noise according to the shape of the wave packet. This study provides a better understanding of the localization. For instance, the QSD unraveling is known to have good localization properties due to its invariance corresponding, in some sense, to a homogeneous distribution of noise. We have seen that the localization rate can be increased by maximizing the norm of the noise correlation factor and adjusting continuously its phase, this last constraint leading to the introduction of a time dependent unraveling.

Since the new unraveling increases localization it is a good candidate for numerical simulations of quantum trajectories and for the solution of the master equation. A numerical comparison of the wave packet size and fluctuations between QSD and the new unraveling shows that the new unraveling performs better than QSD by stabilizing the size of the wave packet.

In connection with the study of the quantum-classical transition, a recent work by Brun et al 27] has shown that the Quantum Jump unraveling tends to a continuous unraveling. It can be easily seen that this unraveling is a member of the set introduced in the present paper. We have shown that for a simple quantum system subject to dissipation all members of the set of continuous unravelings localize with a minimal rate given by the dissipation rate, making localization a general property valid for all unravelings instead of only some particular ones. 


\section{Acknowledgments}

We thank Gernot Alber, Nicolas Gisin, Ian Percival, Rüdiger Schack and Walter Strunz for stimulating discussions. We acknowledge financial support from the EU under its Human Capital and Mobility Programme.

\section{Appendix A. Properties of the noise correlations}

In the case of a linear combination of two Wiener process $N=2$, the noise term $d \zeta$ is specified by the two complex numbers $\alpha_{1}$ and $\alpha_{2}$ which we write as $\alpha_{1}=\rho_{1} e^{i \phi_{1}}$ and $\alpha_{2}=\rho_{2} e^{i \phi_{2}}$. The noise correlation factor becomes

$$
c=\frac{\sum_{n} \alpha_{n}^{2}}{\sum_{n}\left|\alpha_{n}\right|^{2}}=\frac{\rho_{1}^{2} e^{2 i \phi_{1}}+\rho_{2}^{2} e^{2 i \phi_{2}}}{\rho_{1}^{2}+\rho_{2}^{2}}
$$

Using $R=\rho_{2} / \rho_{1}$ and $\theta=2\left(\phi_{2}-\phi_{1}\right)$, this complex number can be rewritten as

$$
c=e^{2 i \phi_{1}} \frac{1+R^{2} e^{i \theta}}{1+R^{2}}
$$

If $\phi_{1}=0$ and $R$ are kept constant and $\theta$ is varied, the denominator will give a circle in the complex plane, centered at $(1,0)$ and of radius $R^{2}$. The denominator will restrict the circle to be inside the unit disk, centered at the origin. The phase $\phi_{1}$ produces only a rotation around the origin. Thus the number $c$ can take any value inside the unit circle only: $|c| \leq 1$. Furthermore, this result can be generalized without difficulty for an arbitrary number $N$ of noise terms.

\section{References}

[1] Louisell W 1973 Quantum Statistical Properties of Radiation (John Wiley and Sons, New York)

[2] Cohen-Tannoudji C, Dupont-Roc J and Grynberg G 1992 Atom-Photon Interactions: Basic Process and Appliactions (John Wiley and Sons, New York)

[3] Gardiner C W 1991 Quantum Noise (Springer, Berlin)

[4] Percival I C Quantum State Diffusion book in preparation.

[5] Zoller P and Gardiner C W 1997 to appear in Elsevier Science Publisher B.V.

[6] Carmichael H 1993 An Open Systems Approach to Quantum Optics, Lecture Notes in Physics m18 (Springer, Berlin)

[7] Gisin N 1989 Helv. Phys. Act. 62363

[8] Gisin N and Percival I C 1992 J. Phys. A: Math. Gen. 255677

[9] Percival I C 1994 J. Phys. A: Math. Gen. 271003

[10] Diósi L 1988 Phys. Lett. A 132233

[11] Halliwell J and Zoupas A 1995 Phys. Rev. D 527294

[12] Zoupas A 1996 Phys. Lett. A 219162 
[13] Strunz W T and Percival I C The semiclassical limit of quantum state diffusion - a phase space approach, submitted to J Phys A

[14] Wiseman H M and Milburn G J 1993 Phys. Rev. A 471652

[15] Dalibard J, Castin Y and Mølmer K 1992 Phys. Rev. Lett. 68580

[16] Gardiner C W, Parkins A S and Zoller P 1992 Phys. Rev. A 464364

[17] Mølmer K, Castin Y and Dalibard J 1993 J. Opt. Soc. Am. B 10524

[18] Castin Y and Mølmer K 1995 Phys. Rev. Lett. 743772

[19] Naraschewski M and Schenzle A 1995 Zeit. Phys. D 3379

[20] Mølmer K and Castin Y 1996 Quantum Semicl. Opt. 849

[21] Nielsen M A 1996 Quantum Semiclass. Opt. 8237

[22] Holland M, Marksteiner S, Marte P and Zoller P 1996 Phys. Rev. Lett. 763683

[23] Steimle T, Alber G and Percival I C 1995 J. Phys. A: Math. Gen. 28 L491

[24] Schack R, Brun T A and Percival I C 1995 J. Phys. A: Math. Gen. 285401

[25] Schack R, Brun T A and Percival I C 1996 Phys. Rev. A 532694

[26] Schack R and Brun T A 1997 Computer Physics Communications, accepted for publication.

[27] Brun T A, Gisin N, O'Mahony P F and Rigo M 1997 Phys. Lett. A 229267

[28] Garraway B and Knight P L 1994 Phys. Rev. A 491266

[29] Garraway B and Knight P L 1994 Phys. Rev. A 502548

[30] Garraway B and Knight P L 1996 Opt. Communic. 123517

[31] Rigo M, Alber G, Mota-Furtado F and O'Mahony P F 1997 Phys. Rev. A 551665

[32] Gisin N and Percival I P Private communication

[33] Gardiner C W 1990 Handbook of Stochastic Methods (Springer-Verlag)

[34] Gisin N 1990 Helv. Phys. Act. 63929

[35] Rigo M and Gisin N 1996 Quantum Semiclass. Opt. 8255

[36] Muñoz-Tapia R 1993 Am. J. Phys. 611005

[37] Hazegawa H and Ezawa H 1980 Progr. Theo. Phys. Suppl. 6941

[38] Spiller T P and Ralph J F 1994 Phys. Lett. A 194235

[39] Gisin N and Rigo M 1995 J. Phys. A: Math. Gen. 287375 